\title{
Surgical technique of using absorbable, knotless, unidirectional barbed suture as a modified purse string suture
}

\author{
Thirugnanam Agasthian \\ Mt Elizabeth Medical Center, Singapore, Singapore \\ Correspondence to: Thirugnanam Agasthian. \#14-12 Mt Elizabeth Medical Centre, 3 Mt Elizabeth., Singapore 228510, Singapore. \\ Email: t.agasthian@gmail.com.
}

\begin{abstract}
Chest tubes play a very important role in thoracic surgery in the drainage of fluid and air from the pleural cavity. Potential major complications during removal of chest tubes are pneumothorax, hemothorax, and pleural fluid leakage. To prevent this usually a purse string or horizontal mattress suture is usually placed at the time of surgery to aid skin closure and healing after chest tube removal. The purpose of this suture is to prevent atmospheric air re-entry during drain removal and fluid leakage after removal. Unfortunately purse string suture can cause pain, infection, unsightly scar, suture breakage and the need for future suture removal increasing cost and burden for the patient. Alternatively, the chest tube can be placed by a subcutaneously tunnelled incision without the need for a purse string suture. In this technique the surrounding subcutaneous tissues spontaneously closes around the chest tube tract during removal due to the valve effect of the tunnelled tract thus avoiding the need for purse string sutures. Tunnelling however causes more tissue trauma and pain and is not possible in modern thoracic surgery where the majority of surgeries are done minimally invasively where the utility incisions are placed directly rather than not tunnelled. To address these problems the use of barbed sutures as a modified chest tube purse string suture is described.
\end{abstract}

Keywords: Chest tube; purse string; barbed sutures

Received: 08 July 2020; Accepted: 08 January 2021; Published: 20 July 2021.

doi: 10.21037 jovs-20-148

View this article at: http://dx.doi.org/10.21037/jovs-20-148

\section{Introduction}

Chest tubes play an important role in thoracic surgery for the drainage of fluid and air from the pleural cavity. Removal of chest tubes can cause pneumothorax, hemothorax and fluid leakage (1-3). To prevent this a nonabsorbable purse string horizontal mattress suture is usually placed at the time of surgery to aid skin closure after chest tube removal $(4,5)$. This suture helps to prevent atmospheric air re-entry during drain removal and fluid leakage after removal. The purse string suture however can cause pain, infection, unsightly scar, and the need for suture removal increasing cost and burden for the patient. To avoid these complications a novel technique using a single continuous absorbable knotless barbed suture to close the skin wound and as a purse string was described (6). With this technique the need for additional assistance during removal, for knotting and tightening of the suture after chest tube removal and for future suture removal were avoided. There was also better healing of the wound with superior cosmesis due to absence of wound tension and foreign body reaction. There were however some disadvantages associated with is technique. In this technique the muscle layer and main skin incision were closed first making subsequent purse string placement around the chest tube difficult due to limited space and especially if the chest drain size is large $(>24 \mathrm{~F}$ ). As anchoring of the chest tube is done last as it can cause accidental dislodgement or displacement. Due to the skin and purse string suture being a single continuous strand, tightening of the suture during removal can cause suboptimal closure of skin edges over the chest tube site especially if the wound is long. Overzealous tightening of the suture can cause pain, excessive tension and puckering of the whole skin wound with inferior cosmesis. To address 


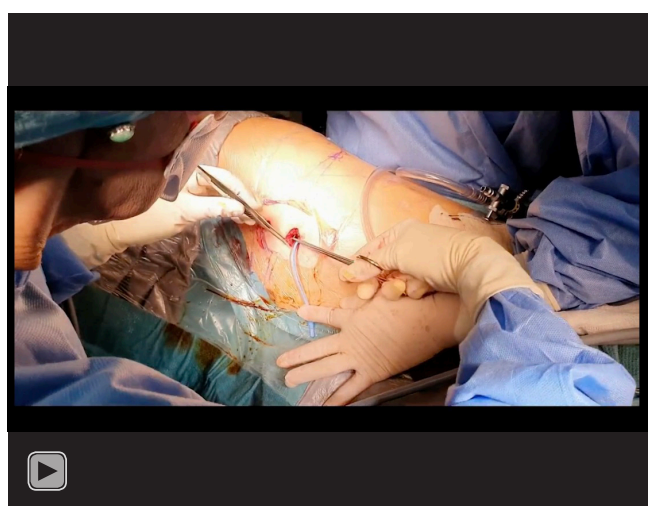

Video 1 Technique of using barbed suture as purse string suture for chest tube placement.

some of these flaws a modified technique is described.

\section{Surgical techniques (video 1)}

(I) A single absorbable 30 Monocryl (Stratafix, Ethicon, Somerville, NJ) or 30 Monoderm (Quill B Braun) unidirectional barbed suture is used for purse string and skin closure.

(II) The purse string suture is anchored to the adjacent muscles and subcutaneous tissues first near the skin exit point of the chest tube. This provides a stable, strong and water tight anchoring closure around the cheat tube site. This helps prevent the ingress of air during removal of chest tube and the leakage of fluid after chest tube removal which can be a problem in VATS when the chest tube is often placed through the un-tunnelled utility incision (Video 1, $0.10 \mathrm{~s}-1.14 \mathrm{~s}$ ).

(III) It is continued subcuticularly around the chest tube and existed 1-2 cm out from the skin edge leaving a sufficient length of suture for subsequent tightening during removal. Putting the purse string first before closure of skin and muscle layers provides adequate room for accurate placement of purse string suture around the chest tube site and prevent accidental placement of suture though the chest tube wall (Video 1, $0.16 \mathrm{~s}-2.07 \mathrm{~s}$ ).

(IV) The chest tube is then anchored to the skin with silk 10 suture to prevent displacement or dislodgement (Video 1, $2.09 \mathrm{~s}-3.10 \mathrm{~s}$ ).

(V) The rest of the muscle and subcutaneous layer is closed with Vicryl (polyglactin Ethicon Somerville NJ) 20 suture (Video 1, $3.12 \mathrm{~s}-4.19 \mathrm{~s}$ ).
(VI) The skin is then closed by subcuticular suturing with the remainder of the same barbed suture (Video 1, $4.20 \mathrm{~s}-4.55 \mathrm{~s})$.

(VII) Surgical skin glue (Dermabond, Ethicon) is applied over the wounds with a small light non allergenic dressing applied over the chest drain site (Video 1, $4.56 \mathrm{~s}-5.24 \mathrm{~s})$.

(VIII) Chest tube is removed by the bedside without any assistance by the following steps (Video 1, $5.25 \mathrm{~s}-6.10 \mathrm{~s}$ ).

(i) Division of anchoring silk suture;

(ii) Application of pressure gauze over the chest tube skin exit point;

(iii) Patient instructed to do Valsalva manoeuvre $(7,8)$;

(iv) Rapid removal of tube with gentle tightening of the purse string barbed suture.

\section{Discussion}

Main considerations in chest tube care is to anchor it securely to prevent dislodgement and to remove it without complications (8). Removal of chest tubes can cause pneumothorax, hemothorax and pleural fluid leakage. To prevent this a nonabsorbable horizontal mattress purse string suture is usually placed at the time of surgery to aid skin closure and healing after chest tube removal. This suture helps to prevent atmospheric air re-entry and fluid leakage. The purse string suture however can cause pain, infection, and unsightly scar especially in young females. There is also the need for future suture removal increasing cost and burden for the patient (1-6).

Chest tube removal is usually done by a junior doctor or a nurse assistant frequently in crammed poorly lighted bedside conditions. It can be a challenging task requiring coordination between patient, doctor and nurse failing which may require reinsertion of chest tube. The usual steps involved are dividing of the anchoring suture first, followed by advance pre knotting of the purse string suture, then applying gauze pressure over the tube site followed by asking the patient to do a Valsalva manoeuvre, then pulling the tube out quickly followed by further gauze pressure on the wound while tightening the pre-knotted suture quickly without breakage $(7,8)$. Mistakes can occur at any one of these steps. Accidental division of the purse string suture mistaken for the anchoring suture can occur especially if the 2 sutures are anchored closely together with no colour differentiation. In these circumstances the purse string suture has to be reinserted at the bedside under 
local anaesthesia. Overzealous knotting and tightening of the purse string can cause suture breakage and acute pain causing the patient to suck in atmospheric air. Loosely opposed skin edges can cause pleural fluid leakage $(3,9)$. In all these circumstances the purse string suture has to be reinserted at the bedside under local anaesthesia. To simplify chest tube removal steps some surgeons do not routinely use a purse string for chest tube removal but apply a heavy compressive petroleum buttressed gauze bandage over the wound after chest tube removal, removing it after 48-72 hours (10). To reduce the risk of pneumothorax and fluid leakage using this technique, subcutaneous tunnelling of the chest tube track is done for spontaneous soft tissue closure of the track. Tunnelling however increases intercostal pain and neuralgia. In the era of minimally invasive surgery chest tubes are routinely placed directly through a port or utility incision without tunnelling often requiring a purse string suture. Others have tried to close the wound at the time of chest drain removal which is difficult to do at the bedside causing considerable pain and distress to patient (11).

To simplify and decrease morbidity of chest tube removal, a novel technique using a single continuous knotless barbed suture both as a purse string suture and for skin closure was described (6). With this technique the tube can be removed by the bedside safely and quickly with minimal assistance without any knots and future suture removal. This was possible due to the absorbable, knotless, and self-locking properties of the unidirectional barbed sutures. This technique though excellent has some problems. As anchoring of the chest tube is done last, it can cause accidental dislodgement or displacement of the chest tube. The closure of the skin, subcutaneous and muscle layers are done first making subsequent placement of subcuticular suture around the chest tube difficult due to limited space. Sometimes due to the continuous nature of the purse string and skin closure suture opposition of the chest tube skin edges is suboptimal after chest tube removal especially if the wound is long. Further attempts at tightening of the suture causes puckering of the skin wound.

In the modified technique instead of using the same continuous suture for wound closure and purse string, they are individually closed by separate barbed sutures with different anchoring points. This is done by dividing a single barbed suture into 2 appropriate lengths. Inserting the purse string suture first before the main wound closure allows the chest tube to be anchored early to prevent dislodgement. Importantly it gives better exposure and more room for easy accurate placement of the subcuticular purse string suture especially when using large bore chest tubes. It also helps reduce the risk of accidental chest tube wall injury or inclusion in the suture. This technique provides a tight seal around the chest tube reducing risks of pneumothorax and fluid leakage after removal which can be a problem especially in VATS where the utility incision is not tunnelled. Separating the wound closures also ensures a tension free accurate opposition of the chest tube skin edges without skin puckering during chest tube removal. There is also less pain reducing the risk of atmospheric air re-entry. Using a knotless absorbable barbed suture minimises foreign body reaction causing less infection, better cosmesis and wound healing with little post-operative wound care needed and avoiding future suture removal (12).

\section{Conclusions}

Use of single absorbable knotless unidirectional barbed suture as a purse string provides a simple safe bedside technique for chest tube removal as well as decreasing morbidity for the patient.

\section{Acknowledgments}

Funding: None.

\section{Footnote}

Conflicts of Interest: The author has completed the ICMJE uniform disclosure form (available at https://jovs. amegroups.com/article/view/10.21037/jovs-20-148/coif). TA serves as an unpaid editorial board member of fournal of Visualized Surgery from June 2019 to May 2021. The author has no other conflicts of interest to declare.

Ethical Statement: The author is accountable for all aspects of the work in ensuring that questions related to the accuracy or integrity of any part of the work are appropriately investigated and resolved. All procedures performed in this study were in accordance the ethical standards of the institutional and/or national research committee(s) and with the Helsinki Declaration (as revised in 2013). Written consent was obtained from the patient for the publication of this manuscript.

Open Access Statement: This is an Open Access article 
distributed in accordance with the Creative Commons Attribution-NonCommercial-NoDerivs 4.0 International License (CC BY-NC-ND 4.0), which permits the noncommercial replication and distribution of the article with the strict proviso that no changes or edits are made and the original work is properly cited (including links to both the formal publication through the relevant DOI and the license). See: https://creativecommons.org/licenses/by-nc-nd/4.0/.

\section{References}

1. Chan L, Reilly KM, Henderson C, et al. Complication rates of tube thoracostomy. Am J Emerg Med 1997;15:368-70.

2. Kwiatt M, Tarbox A, Seamon MJ, et al. Thoracostomy tubes: A comprehensive review of complications and related topics. Int J Crit Illn Inj Sci 2014;4:143-55.

3. Satoh Y. Management of chest drainage tubes after lung Surgery. Gen Thorac Cardiovasc Surg 2016;64:305-8.

4. Rashid MA, Wikström T, Ortenwall P. A simple technique for anchoring chest tubes. Eur Respir J 1998;12:958-9.

5. Flege JB Jr. A simple technique of closing thoracostomy

doi: 10.21037/jovs-20-148

Cite this article as: Agasthian T. Surgical technique of using absorbable, knotless, unidirectional barbed suture as a modified purse string suture. J Vis Surg 2021;7:31. incisions. Surg Gynecol Obstet 1967;124:846-7.

6. Kim MS, Cho JH. A new method of chest drain wound closure using knotless suture material. Ann Thorac Surg 2017;103:e93-5.

7. Bell RL, Ovadia $\mathrm{P}$, Abdullah F, et al. Chest tube removal: end-inspiration or end-expiration? J Trauma 2001;50:674-7.

8. Cerfolio RJ, Bryant AS, Skylizard L, et al. Optimal technique for the removal of chest tubes after pulmonary resection. J Thorac Cardiovasc Surg 2013;145:1535-9.

9. Yokoyama Y, Nakagomi T, Shikata D, et al. A novel technique for chest drain removal using a two layer method with triclosan-coated sutures. J Thorac Dis 2017;9:211-3.

10. Smelt JLC, Simon N, Veres L, et al. The Requirement of Sutures to Close Intercostal Drains Site Wounds in Thoracic Surgery. Ann Thorac Surg 2018;105:438-40.

11. Simon RR, Bailey TD Jr, Abraham E, et al. A new technique for securing a chest tube. Ann Emerg Med 1982;11:619-21.

12. Rauch S, Dutly AE. Chest drains: purse string suture 4.0. Gen Thorac Cardiovasc Surg 2020;68:1055-7. 